\title{
Manejo odontológico de pacientes pediátricos comprometidos sistemáticamente. Revisión bibliográfica
}

Acosta de Camargo $M G^{1}$

Bolívar $M^{1}$

Giunta $C^{1}$

Mora $\mathrm{K}^{1}$

\section{Resumen}

El manejo del paciente pediátrico que está afectado por una enfermedad sistémica requiere de una capacitación por parte del odontopediatra. La patología debe ser conocida así como su manejo médico, hallazgos bucales y manejo odontológico, con la finalidad de hacer un correcto abordaje y evitar posibles complicaciones. Objetivo: Recopilar la opinión de diferentes autores en cuanto al manejo odontológico de algunas enfermedades sistémicas. Materiales y método: Revisión bibliográfica realizada por medio de consulta electrónica mediante las bases de datos: Pubmed, Ebsco-search, LILACS, Proquest y
Biblioteca Cochrane Plus. Se presentan los resultados encontrados en la revisión sobre el manejo odontológico en las cardiopatías congénitas y adquiridas, alteraciones hormonales e inmunológicas, y diabetes. Conclusión: Después de esta revisión bibliográfica se puede concluir que la mayoría de los estudios del manejo de la salud dental en pacientes con compromisos médicos son pobres, aun cuando la necesidad es imperativa. Esta población está aún lejos de comprender plenamente la importancia de la salud bucal a menudo por falta de conocimientos.

Palabras clave: Manejo odontológico, paciente pediátrico, compromiso sistémico.

Artigo de revisão

\section{Cuidados odontológicos em crianças com doenças sistêmicas. Revisão da literatura}

\section{Resumo}

O manejo do paciente pediátrico que apresenta alguma doença sistêmica requer treinamento por parte do odontopediatra. A doença deve ser conhecida e seu tratamento médico, achados bu- cais e cuidados odotológicos, a fim de fazer uma abordagem correta e evitar possíveis complicações. Objetivo: Reunir os pontos de vista de diferentes autores sobre a cuidados odontológicos relacionados a algumas doenças sistêmicas. Materiais e métodos: Uma revisão da literatura 
realizada por consulta eletrônica utilizando bases de dados PubMed, Ebsco-search, LILACS, Cochrane Library e Proquest. São apresentados os resultados encontrados na revisão sobre os cuidados odontológicos em cardiopatias congênitas e adquiridas coração, distúrbios hormonais e imunológicas e diabetes. Conclusão: Após esta revisão de literatura, pode-se concluir que a maioria dos estudos sobre os cuidados com a saúde bucal em pacientes com comprometimento sistêmico são pobres, mesmo quando a necessidade é imperativa. Essa população ainda está longe de compreender plenamente a importância da saúde bucal, muitas vezes, devido à falta de conhecimento.

Palavras-chave: cuidado odontológico, paciente pediátrico, doenças sistêmicas.

Review article

\section{Dental management for children with systemic diseases. Literature review}

\begin{abstract}
The management of a pediatric patient who is suffering a systemic disease requires training by the pediatric dentist. The pathology of the disease must be known and also their medical management, oral findings and dental management, in order to make a correct approach and avoid possible complications. Objetive: to gather the views of different authors regarding the dental management of some systemic disease. Materials and method: A literature review was done by electronic research with the data-base: Medline PubMed, Ebsco-search, LILACS, Proquest y Cochrane Plus Library. It is present the results found of the review about the dental management in congenital and acquired heart disease, hormonal and immune disorders, and diabetes. Conclusion: After this literature review it can be concluded that most studies of the management of dental health in patients with medical commitments are poor, even when the need is imperative. This population is still far from fully understanding the importance of oral health often due to lack of knowledge.
\end{abstract}

Key words: Dental management, pediatric patient, systemic disease.

\section{Introducción}

Cada día la relación entre la salud integral y la salud bucal adquiere mayor importancia. Se sabe que un paciente comprometido sistémicamente puede poner en riesgo su vida si presenta complicaciones bucales como focos infecciosos o mal manejo odontológico. El odontopediatra debe estar capacitado con conocimiento actualizado de las enfermedades que afectan al paciente pediátrico, así como su manejo odontológico, no sólo con respecto a la etiopatología de la enfermedad sino también en cuanto al manejo médico, tratamiento, interacciones farmacológicas, posibles complicaciones y forma de abordaje odontológico. Este tipo de paciente requiere un cuidado mayor ya que su riesgo de compromiso está aumentado. Las interconsultas con médicos deben ser correctamente canalizadas, buscando el trabajo en equipos multidisciplinarios efectivos. Los resultados de esta revisión bibliográfica 
ofrecen una guía para el especialista en odontopediatría que ayude a tales fines.

\section{Lineamientos para el manejo odontoló- gico de los pacientes con cardiopatías congénitas y adquiridas}

Las enfermedades cardíacas se clasifican en dos grupos: Congénitas y Adquiridas. La cardiopatía congénita (CC) es una alteración en la anatomía y/o función del corazón que se desarrolla durante la gestación y que está presente al nacer, independientemente de que sea diagnosticada o no al nacimiento(1). Su origen puede ser genético, ambiental y multifactorial (2). Genético: anemias hereditarias, Hemofilia, Distrofia muscular de Duchene, Síndrome de Hunter, Trisomía 21, Trisomía 18, Trisomía 13-15, XO (Turner) (3-4). Entre las causas ambientales: enfermedades maternas como diabetes, enfermedades del colágeno, fenilcetonuria o las causadas por teratógenos entre ellos químicos como trimetadiona, ácido retinoico, alcohol, físicos como radiaciones, hipoxia, infecciosos como toxoplasmosis o rubeola, mecánicos por compresión intrauterina, y otros como disolventes y pinturas(5). Los multifactoriales se originan por factores ambientales y genéticos combinados (4).

\section{Clasificación de las Cardiopatías Con- génitas según Michelle en el 2009}

1. Lesiones de cortocircuito de izquierda a derecha o Acianótica: Defecto del tabique auricular, Defecto septal ventricular, Defecto septal auriculoventricular, Conducto arterioso persistente.

2. Cortocircuito de derecha a izquierda o Cianótica: Tetralogía de Fallot, Atresia pulmonar, Atresia tricúspide, Anomalía de Ebstein.
3. Derivaciones complejas: Transposición de grandes arterias, Tronco arterioso, Total de drenaje venoso pulmonar anómalo, doble salida del ventrículo derecho e izquierdo. Síndrome del corazón hipoplásico.

4. Lesiones obstructivas: Coartación de la aorta, Interrupción del arco aórtico, estenosis aórtica, estenosis pulmonar (6).

\section{Presentación clínica}

Depende del tipo de cardiopatía, pero en la mayoría de los casos pueden encontrarse disnea, taquicardia, taquipnea, cianosis de piel y mucosas, policitemia, mareo o síncope, aspecto facial rubicundo y soplos (7-8). Retardo en el crecimiento y desarrollo, concentraciones altas de hemoglobina y hematocrito, trombocitopenia, cantidades de fibrinógeno disminuidas y cambios en los tiempos de coagulación (9). Acropaquias (dedos en palillo de tambor), uñas convexas con pérdida del ángulo entre la uña y el lecho ungeal y sudor en la frente como mecanismo compensador de la baja actividad del gasto cardíaco (10), cianosis de mucosas, principalmente en encías, mucosa alveolar y paladar, retardo en la erupción dental, defectos del esmalte, caries dental (11).

\section{Cardiopatías adquiridas \\ Endocarditis Infecciosa (EI)}

Las lesiones endoteliales del corazón o de los vasos ocasionan trombos locales que pueden infectarse a partir de bacterias $u$ hongos que pasan al torrente sanguíneo (endocarditis, endarteritis, flebitis), esto lo hacen especialmente los gérmenes con gran adherencia por los endotelios, como ciertos Streptococos (S) de la cavidad bu- 
cal (12). El S. Viridans, gérmen menos virulentos, con signos y síntomas que simulan variadas patologías, retrasándose significativamente su diagnóstico y el S. aureus (13).

Según los lineamientos de la American Heart Association (AHA) en el año 2008.

1) La profilaxis antimicrobiana en procedimientos dentales invasivos y quirúrgicos en CC está justificada, pues la EI es rara pero potencialmente mortal (14).

2) Como norma general, la profilaxis está indicada siempre que exista un riesgo importante de infección (15) y procedimientos invasivos (16) que se citan en la tabla 1 y 2 , con una dosis única administrada 30 a 60 minutos previo al procedimiento dental sin necesidad de una segunda dosis (17).
3) Para los pacientes que necesiten administración profiláctica y estén bajo antibioticoterapia a largo plazo con los recomendados para la profilaxis, es mejor seleccionar un antimicrobiano diferente, y no aumentar la dosis del que esté tomando (18). Evitar las cefalosporinas por la posible resistencia cruzada del S. viridans. Si es posible puede retrasarse el procedimiento dental hasta 10 días después de completar el tratamiento en curso, para dar tiempo a que se restablezca la flora oral habitual. Si el procedimiento se debe realizar en este caso, debe ajustarse el horario para que reciba una dosis entre 30 y 60 minutos antes (11).

\section{Protocolo cardiopatías}

Las guías de la Asociación Americana del Corazón sobre la profilaxis para EI en el 2007 concluyeron lo siguiente:

\section{Tabla 1.}

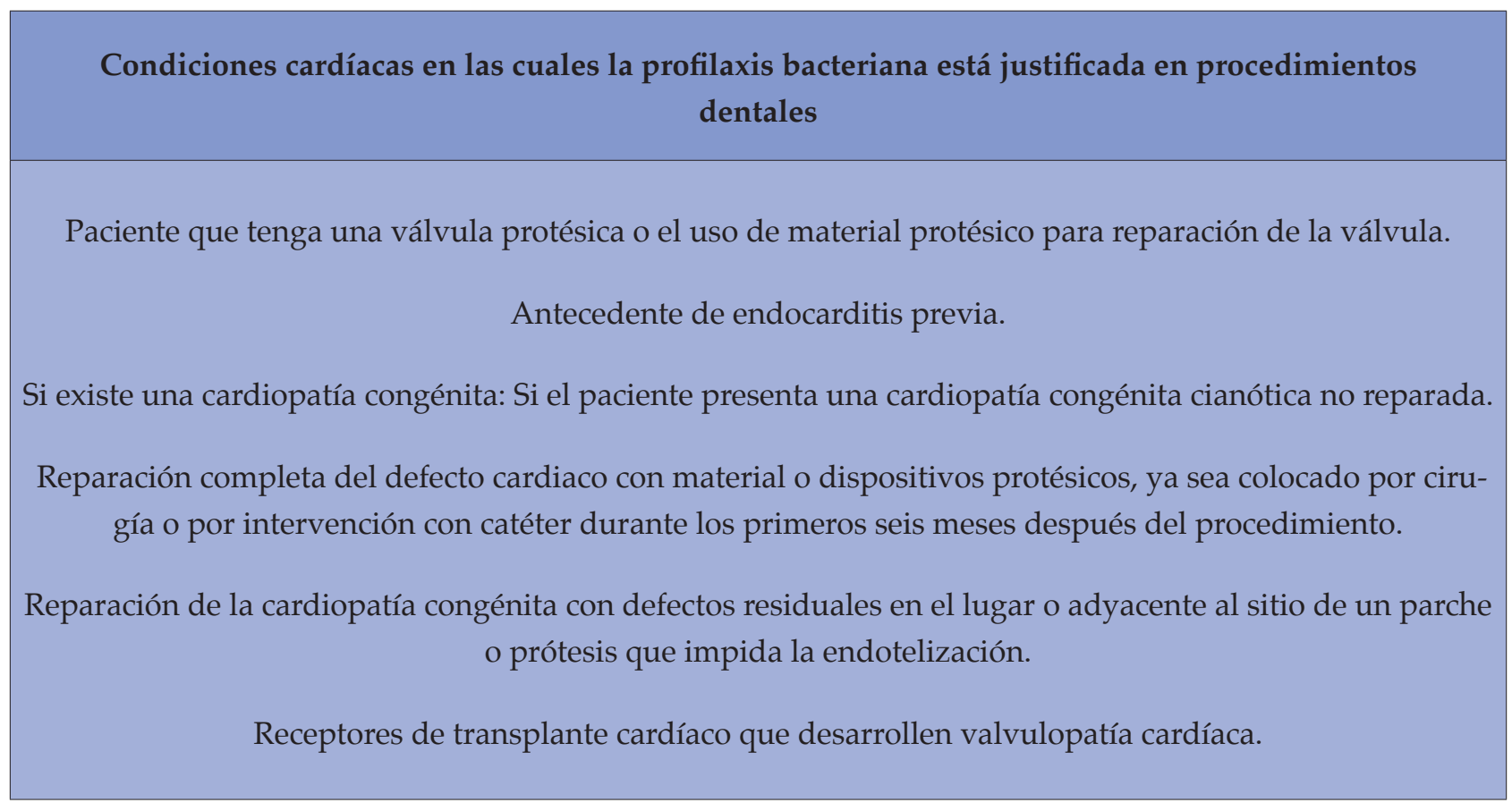

Tomado de: Wilson W et cols. Prevention of Infective Endocarditis. Guidelines From the American Heart Association: A Guideline From the American Heart Association Rheumatic Fever, Endocarditis, and Kawasaki Disease Committee. Research Interdisciplinary Working Group. Circulation. 2007; 116: 1736-54. 
Tabla 2.

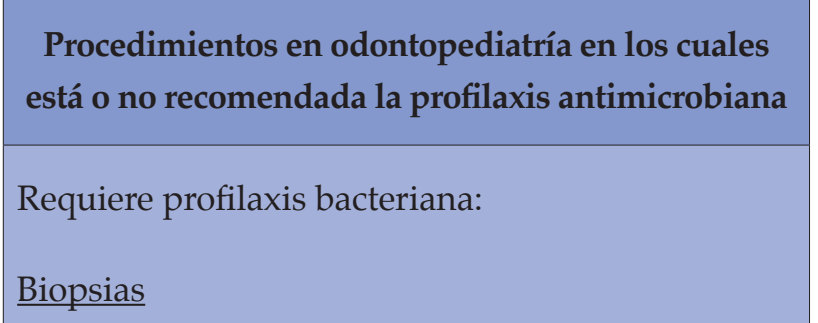

- Extracciones

- Reposición Colocación de bandas para ortodoncia

- Anestesia intraligamentaria

- Cualquier procedimiento del cual se espere sangrado

No requiere profilaxis bacteriana:

- Toma de impresión

- Aplicación de flúor

- Ajuste de aparatos de ortodoncia

- Toma de radiografías

- Operatoria dental

- Colocación de dique de hule

- Anestesia local

Tomado de: Wilson $W$ et cols. Prevention of Infective Endocarditis. Guidelines From the American Heart Association: A Guideline From the American Heart Association Rheumatic Fever, Endocarditis, and Kawasaki Disease Committee. Research Interdisciplinary Working Group. Circulation. 2007; 116: 1736-54.

4) El uso de anestesia con vasoconstrictor no está contraindicado, pero debe ser aplicada con precaución y siguiendo las indicaciones del médico tratante (19).
Los síntomas clásicos incluyen fiebre, anemia, hemocultivos positivos(20). Otros síntomas pueden incluir fatiga, pérdida de peso, sudores nocturnos, anorexia y artralgia. Los émbolos pueden producir dolor torácico, dolor abdominal, ceguera, parálisis y hematuria. Hemorragias de la retina, las petequias pueden ocurrir en tejidos de la piel o de las mucosas y hemorragias lineales pueden ser visibles debajo de las uñas (subcutánea), nódulos de Osler, Janeway (lesiones planas, indoloros, manchas rojas en las palmas y blancas en presión) (14).

\section{Manifestaciones bucales}

Son raras, pueden presentarse piel y mucosas azulosas o de un color rojizo. Si se presenta policitemia pueden encontrarse unas pequenas hemorragias, secundarias a trauma menor de la mucosa oral. Tambien puede haber sangrados gingivales si el paciente esta medicado con anticuagulantes(21).

\section{Manejo odontológico}

Lo más importante en la prevención de la endocarditis infecciosa (EI) antes del procedimiento odontológico parece ser la prevención primaria de las caries (22). Resham menciona que los padres de niños con cardiopatías deben ser educados respecto a la importancia de mantener la higiene bucal en estos niños, los cuales deben recibir atención dental en las clínicas de odontología pediátrica, sobre todo en edades tempranas (23).

Para iniciar el tratamiento odontológico se debe tomar en cuenta:

1) La mayoría de los casos de endocarditis no son atribuibles a un procedimiento invasivo(24). 
Pues sólo el cepillado está asociado con bacteremia en más de uno de cada tres niños (25). Es por ello necesario consultar con el médico tratante sobre el estado bucal y la cobertura profiláctica, pues un número pequeño de EI puede ser prevenida en $100 \%$ con la profilaxis antimicrobiana ya que la mayoría de ellas son causadas probablemente por bacteremias originadas en las actividades cotidianas(11), tales como masticar la comida, cepillarse los dientes, usar hilo dental, uso de palillos de dientes entre las que menciona la Academia Dental Americana (ADA)(26), en concordancia a las pautas dadas por la American Heart Association.

2) El anestésico con epinefrina 1:100.000 no implica riesgos cuando se realiza una buena técnica, solo se requiere de una evaluación cuidadosa de la presión arterial antes del procedimiento, así como tratar de reducir el estrés del paciente durante el tratamiento dental(27), no hay diferencia significativa en el comportamiento de la presión arterial, frecuencia cardíaca, evidencia de isquemia y arritmias con el uso de anestésicos locales con o sin vasoconstrictor, por lo que el uso de epinefrina es seguro(28).

3) El paciente que recibe anticoagulantes y requiere cirugía, al reducir los niveles de este fármaco se puede aumentar el riesgo de tromboembolismo(29), por lo que se recomienda no interrumpir la aspirina(30) ya que las medidas locales son suficientes para controlar el sangrado durante la cirugía. No se debe realizar ningún tratamiento durante procesos infecciosos en fase aguda pues se incrementa el riesgo de hemorragia(31).

4) Los enjuagues antisépticos tópicos son poco eficaces para reducir significativamente la frecuencia, magnitud y duración de la bacteremia asociada con un procedimiento dental ya que no penetran más allá de $3 \mathrm{~mm}$ en el surco periodontal y por lo tanto no llegan donde las bacterias más menudo ganan la entrada a la circulación(32).

5) El odontólogo juega un papel importante eliminando la fiebre, dolor y focos infecciosos de origen dental, mejorando así a la alimentación y la estética(33). Es importante la evaluación precoz de la caries en padres y niños en estos pacientes(34).

\section{Lineamientos para el manejo odontoló- gico de pacientes con Hipertiroidismo e Hipotiroidismo}

La glándula tiroides es un órgano importante del sistema endocrino, localizada en la parte frontal del cuello, debajo de la laringe. Esta glándula libera las hormonas tiroxina (T4) y triyodotironina (T3), las cuales controlan el metabolismo del cuerpo, permiten regular el estado de ánimo, el peso y los niveles de energía tanto físicos como mentales. Los trastornos de la glándula tiroides son resultado de fenómenos autoinmunes (sobreproducción de hormonas o tirotoxicosis) o de procesos que estimulan la destrucción de las células tiroideas (déficit hormonal o hipotiroidismo); además, la glándula tiroidea es un sitio de diversos procesos malignos o benignos (3537). La causa más frecuente de hipertiroidismo en niños es origen autoinmune, y corresponde a un $95 \%$ de los casos de enfermedad de Basedow Graves, caracterizada por bocio, hipertiroidismo y ocasionalmente oftalmopatía (38). El hipotiroidismo es un desorden endocrinológico de naturaleza sistémica, caracterizado por la disfunción de la glándula tiroides que no produce hormonas tiroideas en niveles adecuados. Ocasionalmente, esta disfunción está asociada a la aparición de manifestaciones bucales o altera- 
ciones que comprometen al sistema estomatognático indirectamente (39-41).

Antes de tratar a un paciente con historia de enfermedad tiroidea, el odontólogo debe obtener un correcto diagnóstico con etiología del desorden tiroideo, así como las complicaciones médicas pasadas y la terapia médica recibida. También es de su responsabilidad el manejo del stress, atención a los efectos secundarios de drogas e interacciones aso como la vigilancia de signos y síntomas de toxicidad hormonal (42-43).

\section{Clasificación}

La función tiroidea es dividida en cuatro categorias (44): Hipertiroidismo franco: con TSH sensible indetectable, T4 libre elevada y T3libre elevada. Hipotiroidismo franco: con TSH sensible elevada, T4 libre baja y T3 libre baja. Hipertiroidismo subclínico: con TSH sensible indetectable, T4 libre normal y T3 libre normal. Hipotiroidismo subclínico: con TSH sensible elevada, T4 libre normal y T3 libre normal.

\section{Presentación clinica}

Es de vital importancia que el paciente este controlado sistémicamente, ya que de no ser así, puede desarrollarse una crisis tirotóxica o tormenta tiroidea $(42,45)$. Se debe estar atento ante los problemas de coagulación, así como control de la tensión arterial. También controlar las infecciones por inadecuada cicatrización (42).

\section{Manifestaciones bucales}

Se destacan alteraciones en la formación de dentina, hipoplasia de esmalte, mordida abierta, retraso en la erupción dentaria sin desarrollo radicular, taurodontismo, hipoplasia condilar, atresia maxilar o mandibular, aumento en susceptibilidad a la caries, enfermedad periodontal y gingivitis $(39,46)$, hiposalivación (47-48), disgeusia, macroglosia(49), así como también retraso en la cicatrización de heridas (50-51). Incluyen también alargamiento del tejido tiroideo extra glandular (principalmente en la parte posterior de la lengua), osteoporosis maxilar y mandibular, acelerada erupción dental y síndrome de boca ardiente (52).

\section{Manejo Odontológico}

La combinación de analgésicos que contienen acido acetil salicílico está contraindicada en pacientes con hipertiroidismo porque interfiere con la unión a proteínas de T3 y T4, aumentando su forma libre, esto es peor para la tirotoxicosis (53-54). Los AINES también deben ser usados con cautela, ya que estos pacientes usan B-bloqueantes que pueden disminuir su acción al combinarse con los primeros, por lo tanto el uso de estas drogas debe ser estudiado detalladamente (55-56). El riesgo a crisis por ansiedad esta aumentado. La epinefrina está contraindicada mientras existan signos o síntomas de tirotoxicosis, por lo que el manejo dental debe ser diferido (57-58).

Los fluoruros han sido usados como droga para tratar el hipertiroidismo porque estos reducen la actividad tiroidea efectivamente. Esto se debe a la habilidad del fluoruro de imitar la acción de la tirotropina (TSH). El exceso de flúor esta correlacionado con deficiencia de yodo. El flúor y el yodo, por ser miembros del grupo halógeno de átomos, tienen una relación antagónica. Cuando existe exceso de flúor en el cuerpo pueden interferir en la función de la glándula tiroidea. Es por esta razón, que los fluoruros han estado relacionados con problemas tiroideos. Pacientes 
que quieren evitar el efecto del flúor en su tiroides pueden usar pastas dentales libres de flúor (59-62). Los disturbios causados por flúor son similares a los observados en la deficiencia de yodo(60,63).

Es tarea de los médicos y odontólogos formular el diagnóstico temprano, oportuno y eficaz para canalizar de forma precisa a los pacientes con los especialistas, con objeto de brindar a la población un panorama más amplio de atención interdisciplinaria. El adecuado diagnóstico es indispensable para tener un resultado exitoso(42-43).

\section{Lineamientos para el manejo odontoló- gico de los pacientes con Artritis Juve- nil Idiopática (AJI)}

La AJI es un término que describe clínicamente a un grupo heterogéneo de artritis de causa desconocida y cuyo signo principal es la inflamación crónica de las articulaciones, pero con diferentes categorías dentro de la enfermedad. Su causa es poco entendida y no está disponible ninguna droga que cure la enfermedad, sin embargo, el pronóstico ha mejorado notablemente como resultado de la clasificación y manejo de la enfermedad(64-65).

Se cree que están envueltos factores hereditarios y ambientales y que una reacción autoimmune precipita una cascada de cambios inflamatorios. Una vez que la respuesta immune es iniciada y la inflamación de las articulaciones es disparada, los linfocitos B produces inmunoglobulinas. En algunos sujetos se depositan factores reumatoideos de IgG e IgM en el sinovium(66-67).

La nueva nomenclatura fue enunciada en 1994 por la Liga Internacional de Asociaciones para la Reumatología (ILAR) en un consenso de gru- po donde se estableció el término de Artritis Idiopática Juvenil y una clasificación que pudiera facilitar la comunicación entre especialistas, incluyendo 3 características: Artritis de causa desconocida, evolución de al menos seis semanas y presentación antes de los 16 años(68).

\section{Clasificación}

La nueva clasificación ILAR (Edmonton, 2001) incluye 7 subtipos de AIJ: Artritis sistematica, oligoartritis, poliartritis FR negativo, poliartritis FR positivo, artritis psoriatica, artritis relacionada con entesitis y artritis indeferenciada (68).

\section{Presentación clínica}

Los medicamentos usados en el manejo de la AJI son para controlar los síntomas de dolor, rigidez e inflamación, minimizar la discapacidad funcional y prevenir el daño en la articulación. La mayoría de niños reciben AINE (los más usados son ibuprofen y naproxen), también hay una tendencia a usar inyecciones esteroideas intra-articulares y drogas anti-reumáticas modificadoras de enfermedad (DMARDs) temprano en la enfermedad para minimizar la discapacidad funcional y disminuir el uso de esteroides orales(69-71).

Entre las DMARDs se incluyen el metotrexato y sulfasalazine que han aumentado su uso en reumatología pediátrica, pero que requieren un monitoreo regular. Los efectos secundarios que afecten la boca son raros, pero incluyen estomatitis ulcerativa y parecieran reducirse con el uso de ácido fólico(72-73).

\section{Manifestaciones bucales}

Los pacientes con AJI enfrentan consecuencias que envuelven el sistema musculoesqueletal a 
largo plazo, o casi durante toda su vida. También tienen significativos problemas dentarios, que son prevenibles, pero que requieren una supervisión cercana. Frecuentemente necesitan intervención odontológica y en casos selectos, pueden ser beneficiados con la cirugía ortognática $(65,74)$. La vigilancia farmacológica es esencial para ver la eficacia de tratamiento y los efectos secundarios. La fisioterapia, terapia ocupacional, cuidados de enfermería y apoyo psicológico forman parte del manejo integral de la AJI, así como la incorporación de oftalmólogos, ortopedista y odontólogos al equipo de atención(75-76). En general, estos pacientes reciben medicamentos asociados con aumento en el riesgo de caries (altos en azúcares), lo que hace mayor la aparición de lesiones cariosas. El consumo de metrotreate está posiblemente relacionado con úlceras orales y la ciclosporina, es causa de agrandamientos gingivales(65-67).

Hallazgos clínicos: limitación en apertura de ATM, retrognatia, dificultad en motricidad fina para movimientos como cepillado o uso de hilo dental cuando están afectados los miembros superiores. Cambios salivales: Niveles bajos de calcio, fosforo y potasio, lisosimas e Ig A(66-67).

Se han sugerido ejercicios de aperturas para asegurar la movilidad de la articulación (66-67). En la peor expresión facial de estos pacientes, se puede observar severa retrognatia, mordidas abiertas, microgenia y cara con aspecto de pájaro, con pérdida de dimensión vertical posterior por resorción condilar(65,77).

En pacientes con AJI existen síntomas que incluyen inflamación de ATM con dolor miofacial, así como asimetría, limitación en los movimientos articulares, evidencia radiográfica de asimetría y aplanamiento condilar, además de acentuada muesca antegonial(78).

\section{Manejo odontológico}

En muchos casos se maneja el tratamiento quirúrgico. Se destaca la cirugía de la anquilosis de la ATM, aunque es un tema muy controvertido en la literatura. Se puede practicar una artroplastia simple, una artroplastia con material de interposición (injerto dermo-graso auricular, costocondral y de músculo temporal), sustituir la articulación por prótesis e incluso realizar una distracción ósea mandibular(79). La cirugía correctora de la deformidad dentofacial en un paciente en edad de crecimiento es delicada, pero asociando un injerto costocondral con una osteotomía sagital es una buena solución terapéutica(80). Otra buena opción pudiera ser asociar una artroplastia con músculo temporal en un primer tiempo y una distracción ósea cuatro meses más tarde(79).

Al existir daño en la ATM que ocasiona asimetrías faciales, se sugieren tratamientos con activadores para ayudar al crecimiento de la rama mandibular, para mejorar la función masticatoria y el perfil(81-82). Los tratamientos ortodónticos son necesarios en muchos casos, así como la combinación de los mismos con cirugías ortognáticas $(65,74,82)$.

\section{Lineamientos para el manejo odontoló- gico de pacientes con Diabetes niños y adolescentes}

La diabetes mellitus es un síndrome metabólico que se caracteriza por hiperglucemia con alteración en el metabolismo de los carbohidratos, proteínas y grasas, causada por deficiencia en la producción o en la utilización de la insulina. Es la enfermedad endocrinometabólica más frecuente de la infancia y adolescencia. La diabetes tipo 1 es una condición crónica debido a la 
destrucción de las células beta de los islotes de Langerhans del páncreas por enfermedad autoinmune o sin causa conocida. La hiperglucemia marcada se manifiesta por poliuria, polidipsia, pérdida de peso, a veces con polifagia y visión borrosa. Las consecuencias agudas de la diabetes no controlada que ponen en peligro la vida son la hiperglucemia con cetoacidosis o el síndrome hiperosmolar no cetósico (83-85).

La gran mayoría de los casos de Diabetes se divide en 2 categorías amplias: a) la Diabetes tipo 1, cuya causa es la deficiencia absoluta de la secreción de insulina. Es un proceso autoinmune que ocurre en los islotes pancreáticos con y por marcadores genéticos. B) la Diabetes tipo 2, mucho más frecuente, causada por una combinación de resistencia a la insulina y una respuesta de secreción compensatoria de insulina inadecuada. (85-86).

La diabetes más frecuente en niños es la tipo 1, estos pacientes necesitan la hormona exógena para prevenir la cetoacidosis y conservar la vida. Para poderla diagnosticar, deben presentarse los síntomas clásicos con un nivel aleatorio de glucosa plasmática de $200 \mathrm{mg} / \mathrm{dL}(11.1 \mathrm{mmol} / \mathrm{L}) \mathrm{o}$ más. Si el paciente está asintomático, la concentración plasmática de glucosa en ayuno debe ser de $126 \mathrm{mg}$ / dL (7.0mmol/L) o mayor, o los niveles plasmáticos de glucosa después a las dos horas en una prueba de tolerancia a la glucosa oral deben estar en 200mg/dL o más. Los criterios mencionados en ausencia de síntomas deben confirmarse con una nueva prueba (otro día) $(84,85,87)$. La prueba debe realizarse en un laboratorio que use un método certificado y estandarizado para el ensayo DCCT o FPG $\geq 126 \mathrm{mg}$ / dl. El ayuno se define como la no ingesta calórica por lo menos 8 horas o Glucemia 2 horas posprandial $\geq 200 \mathrm{mg} /$ dl durante la prueba de tolerancia a la glucosa. (87-88).

\section{Clasificación de la Diabetes (Asociación Americana de Diabetes (ADA) en 2012)}

1. La Diabetes tipo 1 se debe a una destrucción de las células beta del páncreas por un proceso autoinmune o de naturaleza idiopática. Se puede presentar a partir de la niñez y la adolescencia en sujetos por lo general delgados; también denominada diabetes del niño y del adolescente. Esta a su vez se sub-clasifica en :

1.1 Diabetes tipo 1 mediada por inmunidad: esta forma de diabetes, que representa solo el 5-10\% de las personas con diabetes, previamente abarcaba los términos diabetes insulinodependiente, diabetes tipo $1 \mathrm{o}$ diabetes de comienzo juvenil, resulta de la destrucción autoinmune de las células $§$ del páncreas $(84,86)$. La diabetes mediada por inmunidad suele ocurrir en la niñez y la adolescencia pero puede ocurrir a cualquier edad, incluso en la octava o novena décadas de la vida $(88,89)$.

1.2 Diabetes Idiopática: algunas formas de diabetes tipo 1 no tienen diagnóstico diferencial. Algunos de estos pacientes tienen insulinopenia permanente y son propensos a la cetoacidosis, pero no tienen evidencia de autoinmunidad. La mayoría son de ascendencia africana o asiática. Esta diabetes tiene un fuerte componente hereditario, carece de pruebas inmunológicas de autoinmunidad de las células $\$$, no están asociadas al HLA y el requerimiento absoluto de terapia de reemplazo de la insulina en los pacientes afectados puede ser intermitente $(86,88,89)$.

2. La Diabetes tipo 2 se produce por defectos en la molécula de insulina o por alteración de sus receptores. La aparición de síntomas es gradual 
en niños mayores y adolescentes por lo general obesos. Habitualmente comienza después de los 30 años y presenta un fuerte componente genético $(85,90)$.

3. Otros tipos específicos: Como defectos genéticos de la función de células $B$, defectos genéticos en la acción de la insulina, enfermedades del páncreas exocrino, endocrinopatías inducidas por fármacos o sustancias químicas.

4. La diabetes gestacional es cuando la intolerancia a la glucosa comienza en el embarazo. Existe riesgo para las madres de desarrollar diabetes tipo 2 en el futuro, así como que los niños padezcan diabetes cuando se conviertan en adultos jóvenes $(84,87,90)$.

5. Otros tipos de diabetes pueden ser: diabetes neonatal o diabetes asociada a otras enfermedades, al uso de ciertos medicamentos, a la edad avanzada $(83,86,88)$.

Los factores ambientales, especialmente las infecciones, están involucradas en la autoinmunidad en la diabetes tipo 1. Las infecciones en ésta y otras enfermedades autoinmunes inducen a lesión tisular e inflamación, provocando la liberación de mediadores químicos proinflamatorios en los tejidos y la producción de proteínas que actúan como autoantígenos, ocasionando reacciones inmunes cruzadas frente a los tejidos propios $(85,89,91)$.

\section{Presentación clínica}

Según la Asociación Americana de la Diabetes (ADA) el tratamiento de la diabetes se basa en el control de la enfermedad y de las complicaciones derivadas de ella. Los objetivos de nuestro tratamiento son: Conseguir niveles adecuado de glucosa en sangre y niveles óptimos de lípi- dos. Dieta adecuada en calorías para conseguir un peso razonable, un crecimiento y desarrollo normal y un embarazo y lactancia adecuados. Evitar sedentarismo y tabaco. Mejorar la salud mediante una nutrición óptima $(88,89,92)$.

Insulina: la insulina regular es de acción rápida y la insulina estándar que se utiliza para corregir rápidamente la hiperglucemia. La mayoría de los niños y adolescentes requieren por lo menos dos inyecciones al día de insulina de acción corta e intermedia para alcanzar un control metabólico satisfactorio; las inyecciones se colocan poco después del desayuno y el almuerzo $(85,87,92)$.

Valor nutricional: la dieta es básica; los niños y adolescentes con diabetes tipo 1 requieren una dieta balanceada con una cantidad adecuada de calorías y nutrientes. En la dieta se recomienda un 55\% de calorías provenientes de carbohidratos complejos, $20 \%$ de proteínas y 30\% de grasas. Deben permitirse golosinas ocasionales dentro del plan dietético, los pacientes y sus familias deben saber cómo ajustar las dosis de insulina en las ocasiones con mayor ingesta calórica, como los días festivos $(86,88,91)$.

Ejercicio: el acondicionamiento físico es importante para los pacientes con diabetes tipo 1, con esto, los requerimientos de insulina serán menores, el control metabólico mejora. Durante los periodos de ejercicio es posible que se necesiten calorías adicionales o menores dosis de insulina para prevenir la hipoglucemia. Cuando el control metabólico es malo, el estrés del ejercicio puede empeorar el control metabólico $(85,89,92)$.

Vigilancia: la glucosa sanguínea se revisa antes de las comidas, antes de las colocaciones (ejemplo: a media mañana, media tarde, al acostarse) y por la noche, alrededor de las $3 \mathrm{am}$, el horario 
del punto más bajo anticipado cuando se utiliza NPH. El control se considera adecuado cuando se obtienen lecturas de glucemia en ayuno y antes de las comidas entre 75 y $150 \mathrm{mg} / \mathrm{dL}$, de 180 a $200 \mathrm{mg} / \mathrm{dL}$ después de las comidas y mayores de 65 a $80 \mathrm{mg} / \mathrm{dL}$ a las 3 de la madrugada $(91,93)$.

Educación: permite que pacientes y familiares comprendan los detalles de la actividad de la insulina, duración y horario, vigilancia de la glucosa en sangre y pruebas para cetonas urinarias. La educación debe ser adecuada según la edad del paciente y nivel educativo de la familia $(84,87)$.

\section{Manifestaciones bucales}

Según la American Academy of Pediatric Dentistry y la American Academy of Pediatrics en 2009, la caries temprana de la infancia ha sido descrita como una enfermedad infecciosa y relacionada a hábitos de dieta inadecuados, por otra parte, la malnutrición por exceso tiene entre sus causas fundamentales los malos hábitos alimentarios, razón por la cual resulta lógico pensar que niños y adolescentes con sobrepeso y con diagnóstico de diabetes presentarán mayor prevalencia de caries dentales (88,94-96).

El aumento sostenido de altas concentraciones sanguíneas de glucosa genera disminución del flujo salival, disminución de la respuesta vascular periférica contribuyendo a la acumulación de placa bacteriana y la formación de tártaro, al desarrollo de caries, halitosis y enfermedad periodontal. Los niños y adolescentes con Diabetes tipo 1 suelen presentar en términos generales mayor respuesta de los tejidos periodontales a la placa bacteriana, lo que se expresa en mayor inflamación gingival y una mayor predisposición a padecer enfermedades periodontales más graves $(86,94,95)$.
Una de las medidas que facilita el control de la enfermedad es el riguroso control de la alimentación basado en el tipo de aporte calórico según la edad, actividad física y el esquema insulínico empleado. Esta alimentación debiera ser controlada en el consumo de carbohidratos, pudiendo actuar como un factor protector contra la caries al considerarse como una dieta poco cariogénica en donde los odontólogos juegan un papel muy importante controlando la alimentación de los pacientes pediátricos (93-95).

Según la Asociación Dental Mexicana desde el año 2003 se creó un protocolo para minimizar el riesgo de emergencias odontológicas en pacientes con diabetes o sospecha de la misma, el odontólogo necesita tener presente ciertas consideraciones antes del inicio del tratamiento dental.

1-Historia Médica: evaluar el control glicémico en la primera cita, junto con los exámenes de laboratorio pertinentes. Dependiendo del propósito y acción a realizar estarán indicados exámenes complementarios en un niño diabético o con sospecha de diabetes, por lo cual disponemos de exámenes diagnósticos y además para control y monitoreo de la siguiente forma: Categóricos: Glucosa 2 horas pospandrial, curva de tolerancia a la glucosa. Intuitivos: Glucosa en sangre en ayunas y glucosa en sangre a las 2 horas. Control y monitoreo: Hemoglobina glucosilada, glucosa en sangre mediante cintas reactivas y curva de tolerancia a la glucosa $(89,92)$.

2-Interacciones Medicamentosas: algunos medicamentos prescritos pueden alterar el control de la glucosa a través de interferencias de la insulina o del metabolismo de los carbohidratos. Por su parte, fármacos como epinefrina, corticoesteroides, fenitoína y drogas que bloquean los canales de calcio, tienen efecto hiperglicemian- 
tes. El odontólogo siempre debe consultar con el médico tratante del paciente (97-98).

3- Horario de Consulta: las citas en la mañana son las recomendables, ya que los niveles endógenos de cortisol son generalmente más altos en este horario. En el caso de los pacientes pediátricos, donde presentan generalmente diabetes insulino-dependiente se debe tener en cuenta el tipo de insulina prescrita y la frecuencia de su aplicación; ya que existen picos de la actividad insulínica, durante los cuales es preferible no efectuar ningún tratamiento odontológico, es decir, las citas deberían ser en el horario donde no coincidan con los picos de actividad insulínica, ya que ese es el periodo de máximo riesgo de desarrollar un episodio de hipoglicemia $(90,94)$.

4- Dieta: el odontólogo debe asegurarse que el paciente haya comido y tomado o aplicado su medicación usual. Si el paciente no desayunó antes de su cita odontológica, pero si se aplicó su dosis normal de insulina, el riesgo de un episodio hipoglicémico se incrementa $(92,98)$.

5- Monitorear los niveles de glucosa en sangre: dependiendo de la historia médica, régimen de medicación y procedimiento a ser ejecutado, el odontólogo necesita medir los niveles de glucosa en sangre antes de iniciar el tratamiento dental, mediante el uso de un dispositivo electrónico medidor de glucosa. Pacientes con bajo nivel de glucosa en sangre (menor de 70mg/dL) deberían tomar un carbohidrato antes del tratamiento para minimizar el riesgo de un evento hipoglicémico. El odontólogo deberá referir a los pacientes con niveles significativamente elevados de glucosa en sangre para una consulta médica antes de realizar el procedimiento odontológico correspondiente $(84,89)$.
6- Durante el tratamiento: la complicación médica más común de la terapia de diabetes que puede ocurrir en el consultorio odontológico es un episodio de hipoglicemia. Los signos y síntomas iniciales incluyen: cambios de humor, hambre, sed y debilidad. Si no es tratada puede producirse inconsciencia, hipotensión, hipotermia, coma y muerte. Si la hipoglicemia ocurre el odontólogo deberá administrar 15 gramos de carbohidratos por vía oral de acción rápida cada 15 minutos hasta normalizar el azúcar en sangre; luego se examina de nuevo los niveles de azúcar en sangre, si esta no se normaliza entonces el odontólogo debe buscar asistencia médica de inmediato $(90,96)$.

7-Después del tratamiento: el odontólogo debe tomar en cuenta las consideraciones post-operatorias en pacientes pediátricos con diabetes, ya que están bajo riesgo de desarrollar infecciones y retardo en la cicatrización. Los pacientes diabéticos tipo 1 y tipo 2 bien controlados, pueden recibir tratamiento odontológico sin necesidad de que haya que modificar el protocolo de atención; sin embargo, si el paciente no está controlado no puede ser atendido odontológicamente $(84,92)$.

La diabetes se asocia a cambios en la cavidad bucal como menor flujo y diferente composición de la saliva, síndrome de boca ardiente, dificultad en la cicatrización de heridas, descenso de la sensibilidad gustativa, mayor riesgo de padecer enfermedad periodontal, caries dental e infecciones oportunistas como Candidiasis bucal, además de ser un factor predisponente para la aparición de leucoplasia y liquen plano. $(83,91,99,100)$.

En los niños una alta glicemia podría asociarse a una disminución del flujo salival, acúmulo de placa bacteriana y por ende caries dental(61-62). 
En pacientes diabéticos no controlados: Infección o mala cicatrización de heridas. En pacientes controlados con insulina: reacción a la insulina. En pacientes diabéticos, comienzo precoz de las complicaciones relacionadas con el sistema cardiovascular, ojos, riñones y sistema nervioso (insuficiencia renal, ceguera por neuropatía periférica, insuficiencia cardíaca congestiva) $(92,97,101)$.

\section{Manejo odontológico}

En los pacientes diabéticos bien controlados no está indicada ninguna alteración o cambio en el plan de tratamiento por parte del odontólogo, salvo que se presente alguna complicación de la diabetes como: Hipertensión, insuficiencia cardíaca congestiva, infarto al miocardio, enfermedad renal, etc $(93,95)$.

\section{Manifestaciones bucales}

Enfermedad periodontal acelerada, abscesos periodontales, infecciones, deficiente cicatrización de heridas en las mucosas, xerostomía, ulceraciones bucales, candidiasis, mucormicosis, insensibilidad.

\section{Tratamiento de emergencia}

En los pacientes con infecciones agudas en la cavidad bucal: el médico debe aumentar la dosis de insulina en el caso de los pacientes insulinodependientes, si es posible, obtenga una muestra de exudado y realice un antibiograma; después comience con el tratamiento con penicilina. En general, otros problemas de urgencia pueden tratarse como en pacientes normales $(91,94,101)$.

Después de esta revisión bibliográfica se puede concluir que la mayoría de los estudios del manejo de la salud dental en pacientes con compromisos médicos son pobres, aun cuando la necesidad es imperativa. Esta población está aún lejos de comprender plenamente la importancia de la salud bucal a menudo por falta de conocimientos. Existe la necesidad del trabajo colaborativo entre odontopediatras, oncólogos, endocrinólogos y cardiólogos pediátricos para realizar programas bien coordinados y terapéuticas que puedan ser desarrolladas para su aplicación eficaz. No de menor importancia es la educación bucal que se le debe brindar a los cuidadores de los pacientes pediátricos comprometidos sistémicamente, ya que en base a lo que se imparta, se podrán conseguir resultados satisfactorios y disminución de complicaciones que pudieran haber sido prevenidas.

\section{Bibliografía}

1. De Soto JS. Manual para Padres de Niños con Cardiopatía Congénita. IMGRAF S.L. Impreso en España. 2006. Disponible en: http: / / www.secardioped.org/Descargas/PyB/PubSEC_006.pdf.

2. Blanco ME, Almeida S, Russinyoll G, Rodríguez de la Torre G, Olivera H, Medina R. Actualización sobre la cardiogénesis y epidemiología de las cardiopatías congénitas. Rev Med Electrón 2009; 31(3).

3. Ferenz C, Neill CA, Boughman JA, Rubin JD, Brenner JI, Perry LW. Congenital cardiovascular malformations associated with cromosomal abnormalities: an epidemiologic study. J Pediatr 1998; 114:79-86.

4. Hubner G ME. Ramirez R. Nazer J. Malformaciones congénitas. Diagnóstico y manejo neonatal. Santiago de Chile: Editorial universitaria; 2005.

5. Botto LD, Correa A. Decreasing the burden of congenital heart anomalies: an epidemiologic evaluation of risk factors and survival. Progress in Pediatric Cardiology 2003; 18: 111-21. 
6. Michelle C. White. Anaesthetic implications of congenital heart disease for children undergoing non-cardiac surgery. Review Article. Anaesthesia \& Intensive Care Medicine 2009; 10 (10): 504-9.

7. Moss AJ, Adams FH, Emmanovilides GC. Heart disease in infants, children and adolescents. 3a ed. Baltimore: Williams \& Wilkins; 1983.

8. Ayala Curiel J. Cardiología para pediatras de atención primaria. Rev Pediatr Aten Primaria. 2009; 11(Supl 17): S451-6.

9. Martins da Silva V, de Oliveira Lopes MV, Leite de Araujo T. Evaluation of the growth percentiles of children with congenital heart disease. Rev Lat Am Enfermagem 2007;15: 298-303.

10. Myung K Park. Cardiología Pediátrica. 5ª ed. Barcelona: Elsevier Mosby; 2008.

7. Herranz C. Control de los niños con cardiopatía congénita en Atención Primaria. Rev Pediatr Aten Primaria 2009;11 (44):

11. Zavala KB, Vinitzky I, Ramírez D. Manejo estomatológico del paciente pediátrico con cardiopatía congénita. Revisión de la literatura. Univ Odontol 2011; 30(64):57-66.

12. Herranz Jordán B. Control de los niños con cardiopatía congénita en Atención Primaria. Rev Pediatr Aten Primaria 2009;1:639-55.

13. Casabe JI. Endocarditis infecciosa una enfermedad cambiante. Medicina 2008; 68: 164-74.

14. Lam DK, Jan A, Sándor GK, Clokie CM. Prevention of Infective Endocarditis: Revised Guidelines from the American Heart Association and the Implications for Dentists. J Can Dent Assoc 2008; 74(5):449-53.

15. Gutiérrez JL, Bagán JV, Bascones A, Llamas R, Llena J, Morales A, Noguerol B, Planells, Prieto J, Salmerón JI. Documento de consenso sobre la utilización de profilaxis antibiótica en cirugía y procedimientos dentales. Av Odontoestomatol 2006; 22 (1).

16. Ramu C, Padmanabhan TV. Indications of antibiotic prophylaxis in dental practice- Review.Asian Pac J Trop Biomed. 2012; 2(9): 749-754. http:/ / www.apjtb.com/zz/20129/15.pdf

17. Bonow RO, Carabello BA, Chatterjee K, de Leon AC Jr, Faxon DP, Freed MD, Gaasch WH, Lytle BW, Nishimura RA, O'Gara PT, O'Rourke RA, Otto CM, Shah PM, Shanewise JS; American College of Cardiology / American Heart Association Task Force on Practice Guidelines. Focused update incorporated into the ACC/AHA 2006 guidelines for the management of patients with valvular heart disease: a report of the American College of Cardiology / American Heart Association Task Force on Practice Guidelines (Writing Committee to Develop Guidelines for the Management of Patients With Valvular Heart Disease): Endorsed by Society of Cardiovascular Anesthesiologists, Society of Cardiovascular Angiology and Interventions, Society of Thoracic Surgeons. J Am Coll Cardiol. 2008; 52(13): e1-142

18. Wilson W, Taubert KA, Gewitz M, Lockhart PB, Baddour LM, Levison M, Bolger A, Cabell CH, Takahashi M, Baltimore RS, Newburger JW, Strom BL, Tani LY, Gerber M, Bonow RO, Pallasch T, Shulman ST, Rowley AH, Burns JC, Gardner T, Durack D. Prevention of Infective Endocarditis. Guidelines From the American Heart Association: A Guideline From the American Heart Association Rheumatic Fever, Endocarditis, and Kawasaki Disease Committee. Research Interdisciplinary Working Group. Circulation. 2007; 116: 1736-54.

19. Paz RC. Manejo odontológico en pacientes cardiópatas. Rev Soc Chil Odontoped 2010; 25(1).

20. Little JW, Falace DA, Miller CS, Rhodus NL. Infective endocarditis. Dental management of the medically compromised patient. 6th edition. Toronto: Mosby, Inc.; 2002.

21. Cardenas JD. Odontologia pediatrica. 4ta edic. Medellin: Corporacion para Investigaciones Biologicas;2009.

22. Herranz C. Control de los niños con cardiopatía congénita en Atención Primaria. Rev Pediatr Aten Primaria 2009;11 (44): 639-55.

23. Reshma Suvarna, Kavita Rai, Amitha M Hegde. Knowledge and Oral Health Attitudes among Parents of Children with Congenital Heart Disease. Int J of Clinic Pediatr Dentist 2011;4(1):25-8.

24. Dajani A, Taubert KA, Wilson W, Bolger AF, Bayer A, Ferrieri P, Gewitz M, Shulman ST, Nouri S, Newburger J, Hutto C, Pallasch T, Gage TW, Levison ME, Peter G, Zuccaro G. Prevention of Bacterial Endocarditis: Recommendations by the American Heart Association. JAMA 1997; 277(22): 1794-801.

25. Roberts GJ, Holzel HS, Sury MRJ, Simmons NA, Gardner P, Longhurst P. Dental bacteremia in children. Pediatr Cardiol $1997 ; 18: 24-7$ 
26. American Dental Association. Profilaxis antibiotica. http:// www.ada.org/5847.aspx

27. Conrado VC, de Andrade J, de Angelis GA, de Andrade AC, Timerman L, Andrade MM, Moreira DR, Sousa AG; Sousa EM, Piegas LS. Efeitos cardiovasculares da anestesia local com vasoconstritor durante exodontia em coronariopatas. Arq Bras Cardiol 2007;88(5).

28. Simões NR; Cols. Efeitos do uso da adrenalina na anestesia local odontológica em portador de coronariopatía. Arq Bras Cardiol 2007; 88 (5).

29. Jiménez Y, Poveda R, Gavaldá C, Margaix M, Sarrión G. An update on the management of anticoagulated patients programmed for dental extractions and surgery. Med Oral Patol Oral Cir Bucal 2008;13:176-9.

30. Brennan M, Wynn R, Miller C. Aspirin and Bleeding in dentistry: an update and recommendations. Oral Surg Oral Med Oral Pathol Oral Radiol Endod 2007; 104: 316-23.

31. Benito M, Benito M, Bernardoni-Socorro C, Arteaga-Vizcaino. Manejo odontológico del paciente con terapia antitrombótica. Acta OdontológicaVenezolana 2009: 47(1): 1-11.

32. Oliver R, Roberts GJ, Hooper L. Penicillins for the prophylaxis of bacterial endocarditis in dentistry. Cochrane Database Syst Rev. 2004: CD003813.

33. Rodríguez VM, Durán GA, De la Teja E, Macedo Q A, Osnaya MH. Rehabilitación bucal bajo anestesia general en pacientes cardiópatas. Experiencia de cuatro años en el Instituto Nacional de Pediatría. Acta Pediatr Mex 2012;33(2):71-5. 34. American Academy of Pediatric Dentistry. Guideline on Infant Oral Health Care 2012;6(13):135-40.

35. Philippe J. Grave's disease in 2009. Rev Med Suisse 2009; 5(198): 764-8.

36. Nunes MT. Hormônios tireoidianos: mecanismo de ação e importância biológica. Arq Bras Encodrinol Metab. 2003;47(6):639-43.

37. Roberts CGP, Ladenson PW. Hypothyroidism. The Lancet. 2004;363:793-803.

38. Zimmerman D, Lteif AN. Thyrotoxicosis in children. Endocrinol Metab Clin N Am 1998;27:109-26.

39. Da Silva SG, Cruz V, Goez MG, Matos PG, Tavares RT, Pedreira RL. Perfil sistemico e manifestacoes bucais em pacientes com hipotireoidismo. Rev Cub Estomatol $2012 ; 49(2)$.

40. Douglas N, Olmedo P, Douglas CR, Monte O. Influência do hipotireoidismoinduzido por propiotiuracil na mucosa gengival do rato. Arq Bras Endocrinol Metab 2006;50(5):926-9.

41. Farias RJM, Venancio RA, Camparis CM. Alterações sistêmicas e suas relações com dores orofaciais. Jornal Brasileiro de Oclusão, ATM e Dor Orofacial 2002;2(6):121-6.

42. Chandna S, Bathla M. Oral manifestations of thyroid disorders and its management. Indian J Endocrinol Metab 2011; 15(Suppl 2):S113-6.

43. Ruiz GA, Soto CA, Yerena BB, Robles GC, Martinez RV. Tratamiento odontológico interdisciplinario en un paciente con alteraciones tiroideas. Reporte de caso. Revista Mexicana de Periodontologia 2012;3(1).

44.Consenso colombiano para el diagnostico y manejo de las enfermedades tiroideas. Acta Med Colomb 1998;24:159-74.

45. Little JW. Thyroid disorders. Part I: Hyperthyroidism. Oral Surg Oral Med Oral Pathol Oral Radiol Endod. 2006;101:27684.

46. Farias RJM, Venancio RA, Camparis CM. Alterações sistêmicas e suas relações com dores orofaciais. Jornal Brasileiro de Oclusão, ATM e Dor Orofacial. 2002;2(6):121-6.

47. Lima FO, Ramalho MJP, Rummler MCO, Rodriguez RTT. Hipotireoidismo altera secreção e composição salivar em ratos sépticos. Rev odonto ciênc 2008;23(1):53-7.

48. Rodriguez TT, Dantas VTA, Ramalho MJP. Participation of nitric oxide synthase and cyclooxygenase-2 in the salivary secretion of hypothyroid endotoxemic rats. Rev odonto ciênc 2009;24(4):383-8.

49. Pinto A, Glick M. Management of patients with thyroid disease: oral health considerations. JADA 2002;133(7):849-58.

50. Biondo-Simões MD, Ioshii SO, Borsato KS, Zimmermann E. O processo de cicatrização influenciado pelo hipotireoidismo e pelo envelhecimento. Estudo da cicatrização da parede abdominal, em ratos. Acta Cirúrgica Brasileira. 2005;20:120-8. 51. Zimmermann E, Ribas-Filho J, Malafaia O, Ribas C, Nassif P, Stieven Filho E, et al. Tracheal suture in rats with hypothyroidism. Wound healing study. Acta Cirúrgica Brasileira. 2009;24(4): 282-9. 
52. Poumpros E, Loberg E, Engstrom C. Thyroid function and root resorption. Angle Orthod 1994;64:389-94.

53.Huber MA, Terezhalmy GT. Risk stratification and dental management of the patient with thyroid dysfunction. Quintessence Int. 2008;39:139-50.

54. García de Francisco S, Taboada Taboada M, Álvarez Vega P. Disfunción tiroidea subclínica. Medifam 2001;11(5): 21-38.

55. Mito RS, Yagiela JA. Hypertensive response to levonordefrin in a patient receiving propranolol: Report of a case. J Am Dent Assoc. 1988;116:55-7.

56.Pérez RA, López MA, Grau LI. Antiinflamatorios no esteroideos (AINES): Consideraciones para su uso estomatológico. Rev Cubana Estomatol 2002; 39(2):119-38.

57.Yagiela JA. Adverse drug interactions in dental practice: Interactions associated with vasoconstrictors. Part V of a series. J Am Dent Assoc. 1999;130:701-9.

58. Pipa VA, García-Pola VM. Anestésicos locales en odontoestomatología. Med oral patol oral cir bucal 2004; 9(5): 438-43. 59. Galletti PM, Joyet G. Effect of fluorine on thyroidal iodine metabolism in hyperthyroidism. J Clin Endocrinol Metab 1958;18:1102-10.

60. Zhan X-A, Li J-X, Wang M, Xu Z-R. Effects of fluoride on growth and thyroid function in young pigs. Fluoride. 2006;39:95-100.

61. Desai VK, Solanki DM, Bansal RK. Epidemiological study of Goiter in endemic fluorosis district of Gujarat. Fluoride. 1993;26:187-190.

62. Susheela AK, Bhatnagar M, Vig K, Mondal NK. Excess fluoride ingestion and thyroid hormone derangements in children living in Delhi, India. Fluoride. 2005;38:98-108.

63. Hosur MB, Puranik RS, Vanaki S, Puranik SR. Study of thyroid hormones free triiodothyronine (FT3), free thyroxine (FT4) and thyroid stimulating hormone (TSH) in subjects with dental fluorosis. Eur J Dent 2012; 6(2):184-90.

64.Jordan A, McDonagh JE. Juvenile idiopathic arthritis: the paediatric perspective.Pediatr Radiol 2006; 36(8):734-42.

65.Barr T, Carmichael NM, Sandor GK. Juvenile idiopathic arthritis: a chronic pediatric musculoskeletal condition with significant orofacial manifestations. J Can Dent Assoc 2008; 74(9):813-21.

66. Ravelli A, Martini A. Juvenile idiopatic arthritis. Lancet 2007;369 (9563):767-78.

67. Murray KJ, Luyrink L, Grom AA, Passo MH, Emery H, Witte D et al. Inmunohistological characterizatics of T cell infiltrates in different forms of childhood onset chronic arthritis. J Rheumatol 1996; 23(12):2116-24.

68. International League of Association for Rheumatology Classification of Juvenile Idiopathic Arthritis. Second revision, Edmonton, 2001. J Rheumatol 2004; 31:390-392.

69. El Maghraoui A. Juvenil idiopathic arthritis. Presse Med 2014;43(1):27-33.

70.Walton AG, Welbury RR, Thomason JM, Foster HE. Oral health and juvenile idiopathic arthritis: a review. Rheumatology (Oxford) 2000; 39(5):550-5.

71.Arguedas O. Artritis idiopática juvenil: Una actualización. Rev méd Hosp Nac Niños 2004; 39(1): 24-33.

72.Peiro Callizo M, Casado Casuso S. Boletin de uso racional del medicamento 2006; 14(1):1-11

73. Ortiz Z, Shea B, Suarez $\square$ Almazor ME, Moher D, Wells GA, Tugwell P. The efficacy of folic acid and folinic acid in reducing methotrexate gastrointestinal toxicity in rheumatoid arthritis. A meta-analysis of randomised control trials. $\mathrm{J}$ Rheumatol1998;25:36-43.

74. Pagnoni M, Amodeo G, Fadda MT, Brauner E, Guarino G, Virciglio P, Iannetti G. Juvenile idiopathic/rheumatoid arthritis and orthognatic surgery without mandibular osteotomies in the remittent phase. J Craniofac Surg 2013;24(6):1940-5. 75. McCann LJ, Woo P. Biologic therapies in juvenile idiopathic arthritis: why and for whom? Acta Reumatol Port 2007; 32(1):15-26.

76. Beena JP. Juvenile idiopatic arthritis: review of the literature and case report. J Dent Child (Chic) 2013;80(1):25-30.

77. Kjellberg H. Craniofacial growth in juvenile chronic arthritis. Acta Odontol Scand 1998;56(6):360-5.

78. Abramowics S, Kim S, Susarla HK, Kaban LB. Differentiating arthritic from myofascial pain in children with juvenile idiopathic arthritis: preliminary report. J Oral Maxillofac Surg 2013; 71(3):493-66. Navarro I, Cebrián JL, Chamorro M, 
López-Arcas JM, Sánchez R, Burgueño M. Anquilosis bilateral de ATM en paciente con artritis idiopática juvenil. Rev Esp Cirug Oral y Maxilofac 2008; 30(2): 127-31.

79. Navarro I, Cebrián JL, Chamorro M, López-Arcas JM, Sánchez R, Burgueño M. Anquilosis bilateral de ATM en paciente con artritis idiopática juvenil. Rev Esp Cirug Oral y Maxilofac 2008; 30(2): 127-31.

80. Stringer DE, Gilbert DH, Herford AS, Boyne PJ. A method of treating the patient with postpubescent juvenile rheumatoid arthritis. J Oral Maxillofac Surg 2007;65(10):1998-2004.

81. Farronato G, Carletti V, Giannini L, Farronato D, Maspero C. Juvenile Idiopathic Arthritis with temporomandibular joint involvement: functional treatment. Eur J Paediatr Dent 2011; 12(2):131-4.

82. von Bremen J, Ruf S. Orthodontic and dentofacial orthopedic management of juvenile idiopathic arthritis: a systematic review of the literature. Orthod Craniofac Res 2011; 14(3):107-15.

83. Rotemberg E, Smaisif K. Crecimiento y desarrollo de niños y jóvenes con Diabetes Mellitus tipo I. J de Odontoestomatología 2010; 12(16):87-9.

84. Martinez C,Martinez L,Luzuriaga T. Perfil psicosocial de niños y adolescentes con Diabetes Mellitus. Bol Pediatri J 2002; 42(6):114-9.

85. American Diabetes Association. Diagnosis and classification of diabetes mellitus. Diabetes Care 2006; 29 Suppl 1:S43-8. 86. Imarai C, Aracena K, Contreras D, Caro J. Relación entre hipoacusia y diabetes mellitus tipo 2. Rev de Otorrinolaringología y cirugía de cabeza y cuello 2013; 73(2):136-9.

87. Crocetti M, Barone M. Diabetes en niños. Oski Compendio de Pediatría. 2da Edición. México: Mc Gram Hill Interamericana Editores; 2004.

88. American Diabetes Association. Diagnostico y clasificación de la Diabetes Mellitus. Diabetes Care 2012; 35 Suppl1:25-9.

89. Zarate O,Castellanos J,Diaz L. Examenes de laboratorio auxiliares en el manejo odontológico del paciente diabético. Revista de la Asociación Dental Mexicana (ADM) 2003; 60(3):115-7.

90. Sanchez I,Martinez A. Diabetes: oral and periodontal implications. Av Odontoestomatol J 2009; 25(5):44-7.

91. Rojas J, Zarante I, Vallejo D. Relación causal entre la diabetes materna pre-existente y asociación VACTERL: caso clínico y revisión de la literatura. Revista española de Endocrinologia Pediátrica. 2013; 4(1):122-7.

92. Herrera J, Goday A, Herrera D. Effects of diabetes on periodontal diseases. Avances en Diabetologia J 2013; 29(5):145-50.

93. Willershausen B, Haas G, Krummenauer F. Relationship between high weight and caries frequency in german elementary school children. Ever J Med Res 2004; 9(8):400-4.

94. Miranda X,Troncoso J,Rodriguez C,Aravena P,Jimenez P. Caries e índice de higiene oral en niños con diabetes mellitus tipo 1. Rev Chil Pediatr 2013;84(5):67-70.

95. Rivera L, Torres F, Fierro C. Oral health condition in overweight preschoolers. Int J Odontostomat 2010; 4(3):267-70.

96. American Academy on Pediatric Dentistry and American Academy of Pediatrics. Policy on early childhood caries: classifications, consequences and preventive strategies. J Pediatr Dent 2009; 30(7):40-3.

97. Cardozo E, Pardi G. Consideraciones a tomar en cuenta en el manejo odontológico del paciente con diabetes. Acta Odontológica Venezolana 2003; 41(1).

98. Clarkson JJ, Gluch JL, Roach K, Zhang ZK. Tratamiento de pacientes odontológicos diabéticos: un problema global. Oral Case Report 2005; 15(1).

99. Herrera D, Goday A, Faria R, López A, Rodriguez J, Herrera L. Asociación entre la Diabetes y la enfermedad periodontal. J Periodoncia y osteointegración 2013; 23(3):171-6.

100. Romero R, Villegas F. Diabetes e infección Periodontal. Rev N Odont 2010; 70(2):12-47.

101. Little J, Falace D, Miller C, Rhodus N. Tratamiento Odontológico del paciente bajo tratamiento médico. 5 a ed. Madrid: Ed Mosby; 1998.

Recibido 16/01/2014

Aceptado 03/02/2015

Correspondencia: gabrieladecamargo@yahoo.com 